\title{
Channel Estimation Algorithms for Third Generation W-CDMA Communication Systems
}

\author{
Khalid A. Qaraqe \\ Tality Corporation \\ 555 River Oaks Parkway \\ San Jose, CA 95134 \\ kqaraqe@tality.com
}

\author{
Sonia Roe \\ Cisco Systems, Inc. \\ 170 West Tasman Drive \\ San Jose, CA 95134 \\ sroe@cisco.com
}

\begin{abstract}
In this paper, three approaches for channel estimation method are proposed and analyzed for third generation (3G) Wideband Code Division Multiple Access (W-CDMA) communication systems. The down link $(D L)$ channel estimation schemes researched are to use time multiplexed pilot - data symbols, code multiplexed common pilot channel (CPICH), and to combine the channel estimate results from these two approaches which is called combined channel estimation (CCE). For channel estimates using time multiplexed pilot-data symbols, we propose a new algorithm called Iterative Multi-slot Averaged Linear Interpolation (IMALI). These methods are modeled and analyzed by means of computer simulations under multipath Raleigh fading channel and compared to the ideal channel estimation result. IMALI and CCE schemes show improvement in channel estimation accuracy and BER performance for high Doppler Frequency.
\end{abstract}

\section{Introduction}

Estimating the fading channel is a crucial part of the digital receiver since the decoded data depends on the channel estimation output. In December 1999, Third Generation Project Partnership (3GPP) and UMTS standardization toward IMT-2000 agreed to introduce a common pilot channel (CPICH)[1] with several $\mathrm{dBs}$ stronger than the Dedicated Physical Channel (DPCH). In addition to $\mathrm{CPICH}$, W-CDMA physical channel specification employs coherent detection in the DL by time multiplexing the pilot and data symbols in every time slot. These pilot symbols (referred to as time division multiplexed (TDM) pilot symbols) further aid the receiver in channel estimation, signal to interfernce ratio (SIR) estimation and demodulation of the transmission power control (TPC) bits $[1,2,3,4]$. One feature of the TDM pilot is that the embedded pilots will allow DL adaptive antenna employment at the BS [4]. For High Doppler Frequency channel estimation based only on TDM pilot causes degradation in channel estimates and loss in link margin.

Another thought for channel estimation scheme is using combined algorithm that exploits both IMALI scheme and CPICH [5].

In W-CDMA, symbols are transmitted using Quadrature Phase Shift Keying (QPSK) and Direct Sequence CDMA (DS-CDMA). The chip rate is 3.84 Mcps and each physical channel is organized in a frame structure that consists of 15 slots with 2560 chips in each slot as shown in Figure 1. For DL, pilot symbols are time multiplexed with data symbols, every slot starts with group of pilot symbols $(2,4,8$ or 16 bits) according to the channel rate, which may be used to estimate the channel and to perform synchronization.

The $\mathrm{CPICH}$ is a fixed rate $(30 \mathrm{kbps}$, and spreading factor $\mathrm{SF}=256$ ). Figure 2 shows the frame structure of the CPICH. More detailed structure of DPCH and $\mathrm{CPICH}$ description can be found in [1].

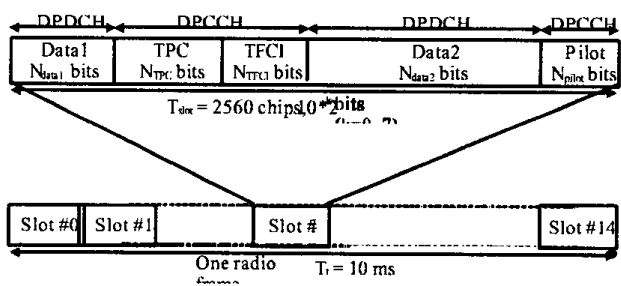

Figure 1: Frame Structure for DPCH 


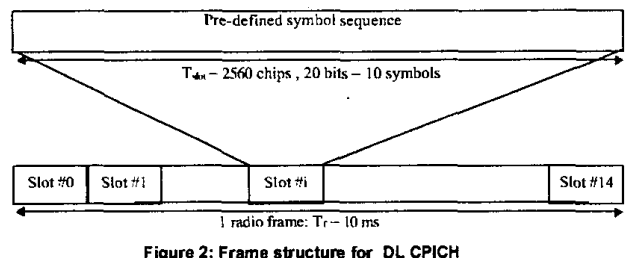

Figure 2: Frame structure for DL CPICH

\section{Channel Estimation Algorithms}

\section{A. IMALI Algorithm}

In order to coherently demodulate the received symbols, the effect of channel rotation has to be removed. The channel estimation algorithm uses time multiplexed pilot symbols to coherently determine the signal phase rotation and fading magnitude caused by propagation through the DL channel. The received signal consists of sequence of slots each containing $N_{p}$ pilot symbols following $\mathrm{N}_{\mathrm{d}}$ data symbols. Iterative Multi-slot Averaged Linear Interpolation (IMALI) algorithm uses the time multiplexed pilot and data symbols in every slot. IMALI technique is implemented by first averaging multiple instantaneous channel estimations without any weighting, and then linearly interpolating current average value with the adjacent average value. The demodulated data is refined in one or more iterations by using both pilot and data symbols $[6,7]$.

The transmitted signal that consists of pilot and traffic channels can be represented by

$$
x(t)=\left(d_{i}+j d_{q}\right) c_{o v s f}\left(s c_{i}+j s c_{q}\right)
$$

Where $d_{i}+j d_{q}$ is the QPSK data symbol, $c_{\text {ovsf }}$ is the channelization code, and $\mathrm{sc}_{\mathrm{i}}+\mathrm{jsc}_{\mathrm{q}}$ is complex scrambling code.

The received signal can be written as

$$
r(t)=x(t) \sum_{i=0}^{L-1} h_{i}(t)+N(t)
$$

Where $h_{i}(t)$ is the complex channel coefficient for $i-t h$ multipath, and $\mathrm{N}(\mathrm{t})$ is complex AWGN noise.
If the m-th pilot symbol in the $\mathrm{n}$-th slot of the $l$-th path is represented as $r_{l}(n, m)$, the average of the pilot symbol $N_{p}$ in the the $n$-th slot, or the instantaneous channel estimation of $\mathrm{n}$-th slot can be expressed as [9]

$$
\hat{\xi}_{l}(n)=\frac{1}{N_{P}} \sum_{m=0}^{N_{p}-1} r_{l}(n, m)
$$

In MALI method, we average three slot instantaneous channel estimations by sliding one slot at a time and interpolating with the previous average as shown in Figure 3. Interpolation values are applied to corresponding data symbols of n-th slot at each symbol rate.

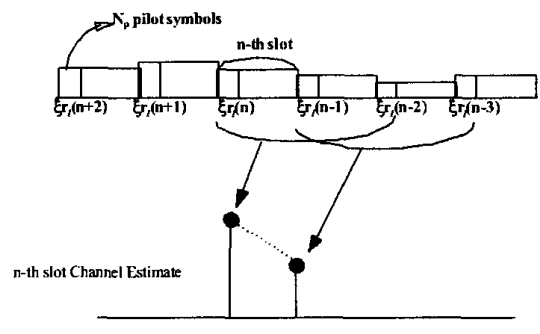

Figure 3: Multi Slot Averaging and Interpolation

The channel estimate of the $j$-th symbol in the n-th slot can be expressed as:

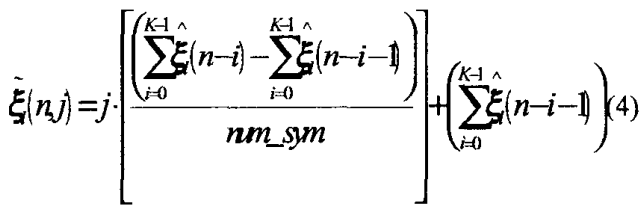

As a second stage of channel estimation, Figure 4 shows IMALI algorithm using the demodulated data from MALI channel estimation output for the initial iteration. For more accuracy the iteration process can be repeated as needed. 


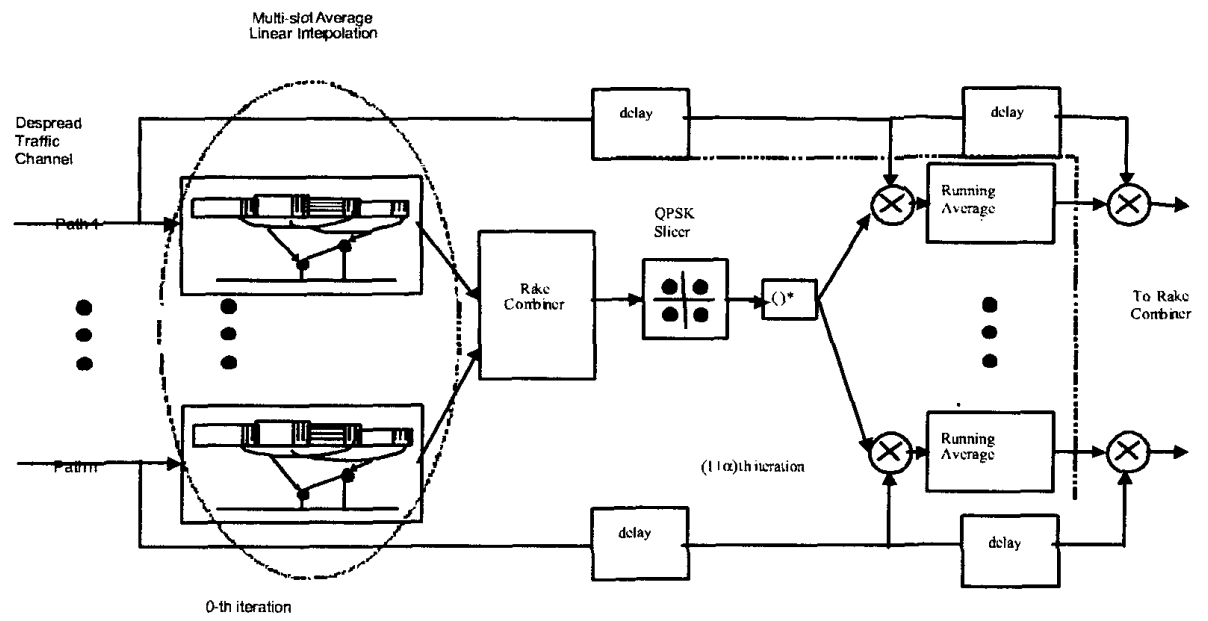

Figure 4: IMALI Channel Estimation Module Scheme

\section{B. CPICH Algorithm}

CPICH is a code multiplexed pilot tone. To estimate channel characteristics from $\mathrm{CPICH}, \mathrm{CPICH}$ is despreaded by corresponding OVSF code with spreading factor of 256 , then multiplied by the complex conjugate of the original pilot pattern. The signal output from this procedure includes random noise. To overcome distortion caused by the random noise, the despread CPICH signal is averaged by moving window average with duration of 6 pilot symbols as shown in Figure 5.

\section{Combined Channel Estimation Algorithm (CCE)}

For combined algorithm, the result from code multiplexed pilot tone, or $\mathrm{CPICH}$ based channel estimation method and time multiplexed pilot symbols, or IMALI method are combined together to make a single channel estimation result.

The channel estimate output from $\mathrm{CPICH}$ and IMALI are combined by ratio of 0.9 to 0.1 with stronger weight applied to $\mathrm{CPICH}$ based channel estimation.

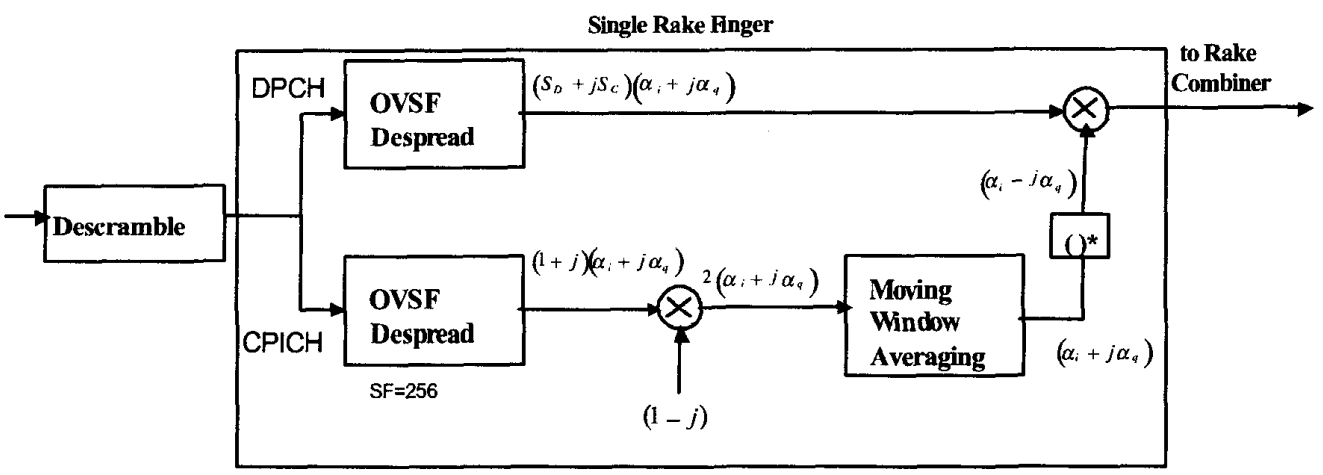

Figure $5 \mathrm{CPICH}$ channel Estimation Module 


\section{Simulation Parameters}

Table 1 shows simulation parameters and conditions that are used for the system modeling and simulations. Case 3 represents multipath Rayliegh fading channel as it is configured in $3 \mathrm{GPP}$-specification. [8]

Table 1 Simulation conditions and parameters

\begin{tabular}{|c|c|c|}
\hline 108 & Descrietan & Soraneters \\
\hline $1 \%$ & Slot format number & 11 \\
\hline 2 & Time tracking & Perfect \\
\hline 3 & Power control & Off \\
\hline 4 & Weights for WMSA & 0.61 .01 .00 .6 \\
\hline$\$$ & $\begin{array}{l}\mathrm{CPICH} \\
\text { interval }\end{array}$ & 6 symbols \\
\hline 菜 & $\begin{array}{l}\text { Iteration parameters } \\
\text { used for ICE and } \\
\text { IMALI and Averaging } \\
\text { window size }\end{array}$ & $\begin{array}{l}20 \text { symbols for } \\
\text { both 1st and 2nd } \\
\text { iteration }\end{array}$ \\
\hline \%. & CPICH Power & $\begin{array}{l}6 \mathrm{~dB} \text { higher than } \\
\text { DPCH }\end{array}$ \\
\hline 8 & Antenna Diversity & One Antenna \\
\hline 10 & Multipath & Case 3 \\
\hline 10 & Number of samples & 10000 \\
\hline 12 & Coding Rate & $1 / 3 . K=9$ \\
\hline 13 & Data Modulation & QPSK \\
\hline 14 & Number of Fingers & 4 \\
\hline 18 & Chip Rate & $3.84 \mathrm{Mchips} / \mathrm{s}$ \\
\hline
\end{tabular}

\section{Simulation Results and Discussion}

Figures 6-9 show the simulation results of the alternative algorithms of the channel estimation for W-
CDMA/3GPP communication systems. MS speeds under test are $100,150,200$ and $250 \mathrm{~km} / \mathrm{h}$. Ideal channel estimation is used as a reference curve to compare BER performance results from different channel estimation methods. For $100 \mathrm{~km} / \mathrm{h}$, the BER performance degradation of $\mathrm{CPICH}$ and the combined algorithms is about $0.5 \mathrm{~dB}$ from the ideal channel estimation at coded BER target of $10^{-3}$. For higher Doppler frequencies, IMALI method outperforms ICE or WMSA [9] algorithms. In case adaptive antenna is employed at the BS, time multiplexed pilot should be used.

Combined algorithm, or CCE presents a better BER performance compared to the other methods, especially at high Doppler frequency. This scheme can be used with or without adaptive antenna.

\section{Conclusions}

Three approaches of the channel estimation algorithms were analyzed and presented in this paper.

A new method, IMALI is introduced which is suitable in case adaptive antennas are employed. The channel estimation performance was improved by using IMALI method compared to conventional WMSA or ICE method, particularly in high Doppler frequencies. IMALI and ICE show approximately $1 \mathrm{~dB}$ gain over WMSA at $100 \mathrm{~km} / \mathrm{h}$ and $150 \mathrm{~km} / \mathrm{h}$. At vehicular speed $200 \mathrm{~km} / \mathrm{h}$, IMALI demonstrates $0.7 \mathrm{~dB}$ gain over ICE. At $250 \mathrm{~km} / \mathrm{h}$, the BER of ICE and WMSA saturates before reaching $10^{-3}$ point, while the saturation point of IMALI is below $10^{-3}$ point.

Combined method is to employ both IMALI and $\mathrm{CPICH}$ with weights applied. The advantage of CCE is to utilize fading channel information from diversified pilot sources enabling adaptive antenna employment as well. Thus, CCE approach is a strong candidate for the practical implementation of the channel estimation. 


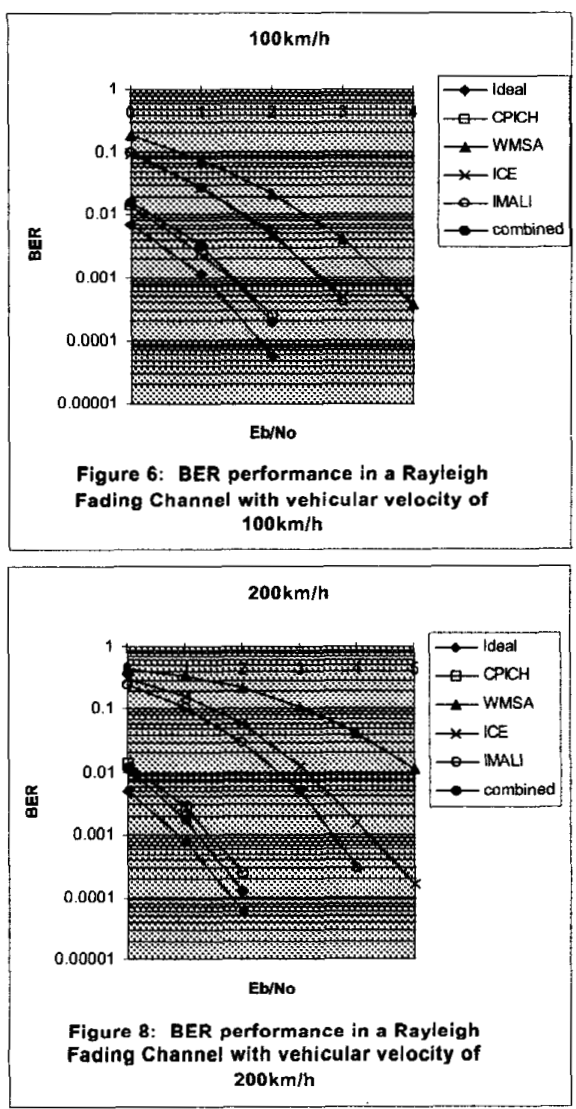

\section{REFERENCES}

[1] 3GPP Technical Specification Group Radio Access Network; Physical Channels and Mapping of Transport Chamels onto Physical Channels (FDD) Specification, 1999 : TS 25.211 V3.1.0 (99-12)

[2] 3GPP Technical Specification Group Radio Access Network; Multiplexing and Channel Coding (FDD) Specification, 1999: TS 25.212 V3.1.0 (99-12).

[3] 3GPP Technical Specification Group Radio Access Network; Spreading and Modulation (FDD) Specification 1999: TS 25.213 V3.1. 0 (99-12)

[4] 3GPP Technical Specification Group Radio Access Network; Physical Channels and Mapping of Transport Channels onto Physical Channels (FDD) Specification, 1999: TS S1.11 V 2.0.0 (99-04).

[5] Seunghyun Min and Kwang Bok Lee, " Channel Estimation Based on Pilot and Data Traffic Channels For DS/CDMA Systems",Global Telecommunications Conference, 1998. GLOBECOM 1998. The Bridge to Global Integration.

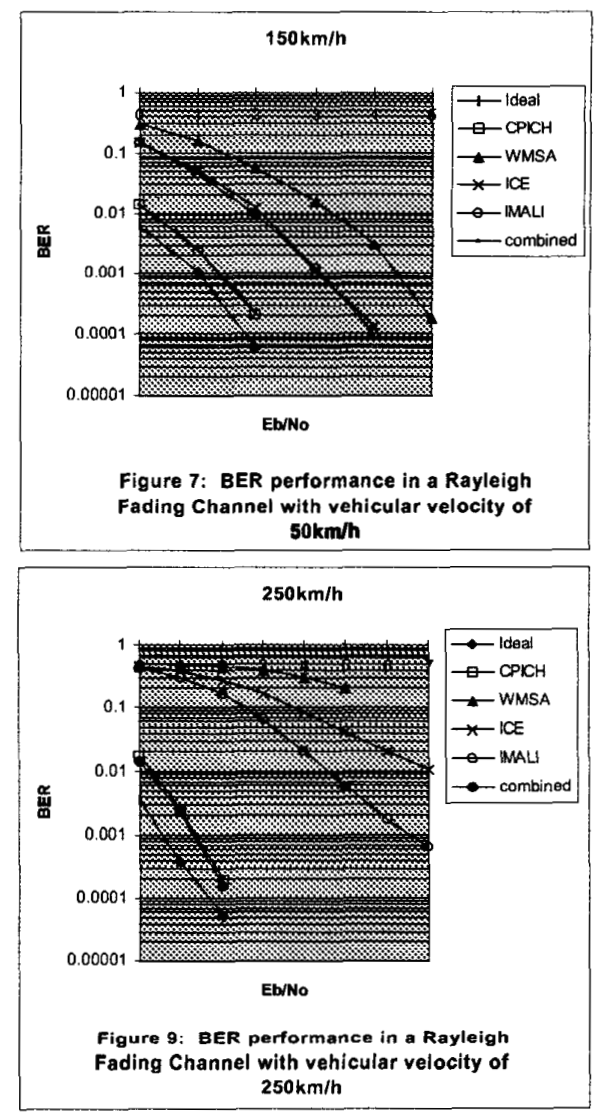

IEEE Volume: 3,1998 , Page(s): $1384-1389$ vol. 3.

[6] ETSI SMG2 UMTS Physical Layer Expert Group, Meeting No. 5, Tdoc SMG2 UMTS-L1 228/98, Gatwick, UK, July 15-17, 1998.

[7] Timothy M. Schmidl, Anand G. Dabak, and Srinath Hoser, "The Use of Itrative Channel Estimation (ICE) to Improve Linke Margin in Wideband CDMA Systems," Vehicular Technology Conference, 1999, IEEE, $49^{\text {th }}$, Volume: 2, 1999, Page(s): $1307-1311$ vol.2.

[8] 3GPP Technical Specification Group RAN WG4, UE Radio Transmission and Reception, 1999, TS 25.101 V3.1.0 (99-12)

[9] H. Andoh, M. Sawahashi, and F. Adachi," Channel Estimation Using Time Multiplexed Pilot Symbols for Coherent Ruke Combining for DS-CDMA Mobile Radio", Personal, Indoor and Mobile Radio Communications, 1997. Waves of the Year 2000. PIMRC '97., The 8th IEEE International Symposium on volume: 3,1997 , Page(s): 954 . 958 vol.3. 